\title{
Quantum Interference as the Source of Steric Asymmetry and Parity Propensity Rules in NO-Rare Gas Inelastic Scattering - Supporting Information -
}

\author{
Arjan Gijsbertsen and Steven Stolte \\ Laser Centre and Department of Physical Chemistry, Vrije Universiteit Amsterdam, \\ De Boelelaan 1083, 1081 HV Amsterdam, The Netherlands \\ Harold Linnartz \\ Sackler laboratory for Astrophysics, Leiden Observatory, \\ P.O. Box 9513, 2300 RA Leiden, The Netherlands \\ Craig A. Taatjes \\ Combustion Research Facility, Mailstop 9055, \\ Sandia National Laboratories, Livermore, CA 94550, USA
}

(Dated: 5th December 2005) 


\section{S.I. COMPLETE REFERENCES}

9 HIBRIDON is a package of programs for the time independent quantum treatment of inelastic collisions written by M. H. Alexander, D. E. Manolopoulos, H.J. Werner, and B. Follmeg, with contributions by P. F. Vohralik, D. Lemoine, G. Corey, R. Gordon, B. Johnson, T. Orlikowski, A. Berning, A. Degli-Esposti, C. Rist, P. Dagdigian, B. Pouilly, G. van der Sanden, M. Yang, F. de Weerd, and S. Gregurick. More information and a copy of the code can be obtained from http://www.chem.umd.edu/physical/alexander/hibridon/.

\section{S.II. THE SIGN OF THE STERIC ASYMMETRY.}

As briefly mentioned before in this work, there still is an important unsolved discrepancy between theory and experiment for collisions of rare gases and NO. There is good agreement as far as the absolute value of the steric asymmetry ratio $(S)$ is concerned, but theory and experiments yield an opposite sign of $S$. In both Ref. 1 and this work, it was decided for now to multiply the experimental values of $S$ with -1 when plotting them in order to enable quantitative comparison. The sign problem for $S$ of collisions with NO is extensively discussed in Refs. 2 and 3. Although not yet published when Refs. 2 and 3 were published, the QQT provides an important argument in discussions on the sign of the steric asymmetry.

All theoretical results for rare gas collisions with oriented $\mathrm{NO}$ and $\mathrm{OH}^{4}$ are, since the revision of Ref. 2, consonant with a so-called classical ball-and-stick model. If a stick is attached to a ball, it is easier to make that system rotate by hitting the stick than by hitting the ball. The torque that can be applied on the stick is larger than on the ball. For Ar colliding with $\mathrm{OH}$ the analogy with the ball (O-end) and stick (H-end) is easily seen. Both experiment and theory shows that highest rotational states are preferentially produced by collisions onto the H-end of the molecule. For NO, the ball-and-stick effect is less pronounced, but it is still there. The N-end extends further from the center of mass than the O-end and therefore it allows for a larger torque. It is expected that relatively high rotational states are preferentially due to N-end collisions. Looking at Fig. 1 of the main document it can easily be seen that this corresponds to theoretical results, while experimental results show that 


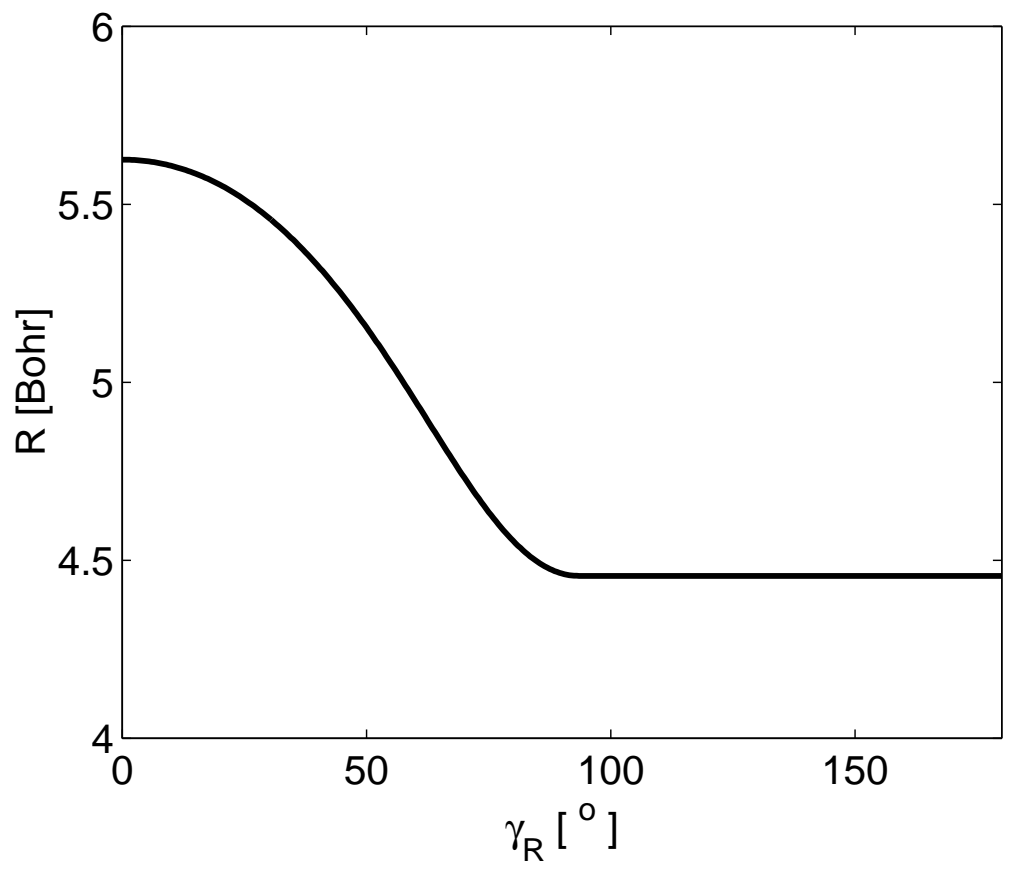

Figure S.1: A hard shell was prepared such that the N-end (around $\gamma_{R}=0^{\circ}$ ) is similar to that used for He-NO collisions, the O-end (around $\gamma_{R}=180^{\circ}$ ) is spherical (represented by a horizontal line). Classically no torque can be applied on the spherical end. From the ball and stick model one expects the $S$ to be overall positive, except for elastic collisions $(\Delta j=0)$ where no torque is necessary.

O-end collisions are preferred for high rotational states. Recall that experimental results in Fig. 1 of the main document are multiplied with a factor -1 .

The ball-and-stick model can be exploited to see whether a sign error has been made in the QQT. It is easy to design a hard shell that has extreme a ball-and-stick features. The outcome of calculations with such a shell should immediately make clear what the orientation is. For such a test, the O-end of the He-NO hard shell was made spherical from the minimum of $R_{s}\left(\gamma_{R}\right)$ on. In Fig. S.1, the adapted hard shell is shown. The original shell is drawn in Fig. 6 of the main document. The steric asymmetry that follows from the QQT calculations is shown in Fig. S.2 and leaves little room for a sign error. Only for elastic collisions $(\Delta j=0)$ is a negative steric asymmetry ratio observed, which means that elastic scattering arises principally from O-end scattering. A positive $S$ is seen for all 


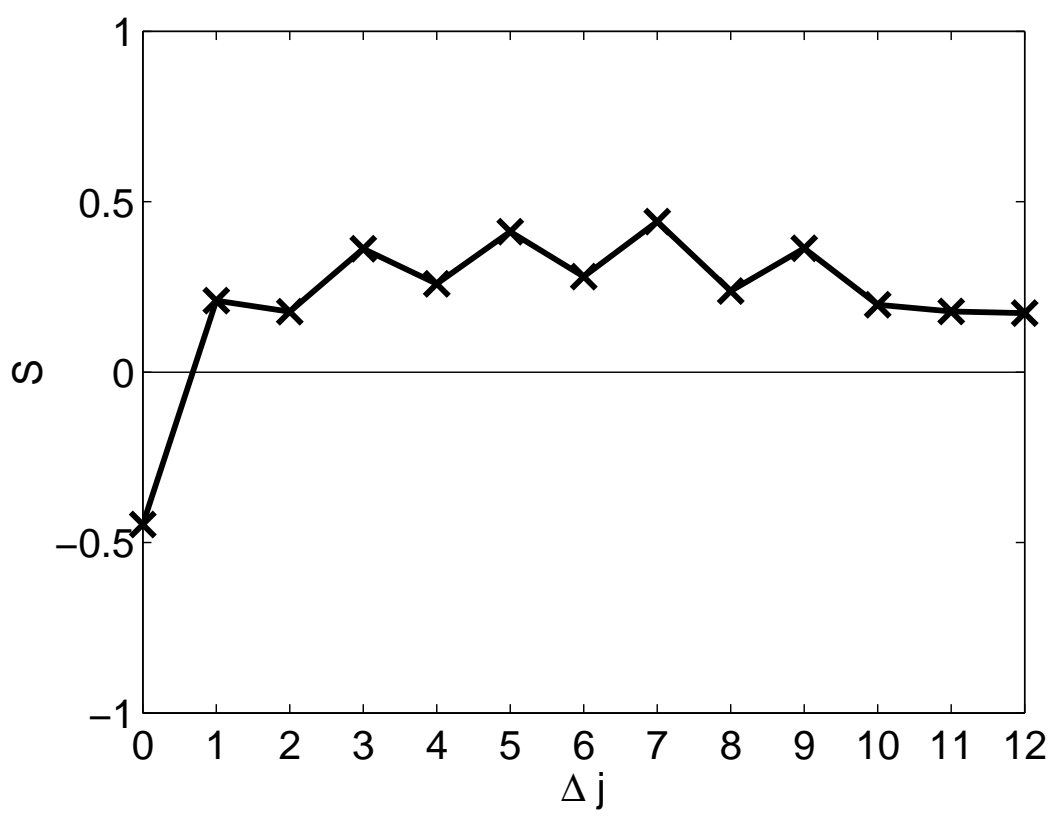

Figure S.2: Steric asymmetry ratio for the specially designed hard shell in Fig. S.1. Classically no torque can be applied on the spherical end. From the ball-and-stick model one expects the $S$ to be overall positive, except for elastic collisions $(\Delta j=0)$ where no torque is necessary. The result for $S$ provides a strong indication that the theoretical sign of $S$ is correct.

inelastic channels $(\Delta j>0)$, which implies that the N-end is preferred. This "ball-and-stick effect" becomes more extreme as the stick is extended farther from the center of mass.

1 M. J. L. de Lange, S. Stolte, C. A. Taatjes, J. Kłos, G. C. Groenenboom, and A. v.d. Avoird, J. Chem. Phys., 2004, 121, 11691.

2 A. Gijsbertsen, M. J. L. de Lange, A. E. Wiskerke, H. Linnartz, M. Drabbels, J. Klos, and S. Stolte, Chem. Phys., 2004, 301, 293.

3 A. Gijsbertsen, H. Linnartz, J. Klos, and S. Stolte, Physica Scripta, 2005, 72, 1-5.

4 M. C. van Beek, J. J. ter Meulen, and M. H. Alexander, J. Chem. Phys, 2000, 113, 637. 